\title{
Legitimación y crítica a la desigualdad: una aproximación pragmática
}

\author{
Legitimation and critique of inequality: \\ a pragmatic approach \\ Virginia Guzmán / virginia.guzman.barcos@gmail.com
Centro de Estudios de la Mujer, Chile \\ Emmanuelle Barozet / ebarozet@uchile.cl \\ Universidad de Chile, Chile \\ María Luisa Méndez / marialuisa.mendez@udp.cl \\ Universidad Diego Portales, Chile
}

\begin{abstract}
Addressing a pragmatic sociology perspective, this article presents the results of a study carried out in Chile in a context characterized by growing public debate about social inequalities and increasing criticisms from ordinary people. Using a methodology based on a classificatory card game, we seek to understand how people describe society, its hierarchies, and the groups that compose it, as well as the arguments used to justify or criticize inequalities. We analyze the principles and values that organize the representations of Chilean society and focus on the dynamics of class and gender that feed those classifications. This exercise helps show that social critique and legitimation of inequality can operate simultaneously but at different levels.
\end{abstract}

Key words: inequality, critique, legitimation, pragmatic sociology, injustice.

Resumen: En el marco de una sociología pragmática, este artículo presenta los resultados de una investigación llevada a cabo en Chile en un contexto caracterizado por un incremento del debate público acerca de las desigualdades sociales y su crítica de parte de las personas comunes y corrientes. Usando una metodología de juegos de clasificaciones de naipes, buscamos entender cómo las personas describen la sociedad, sus jerarquías, los grupos que la integran y con base en qué argumentos justifican las desigualdades o las critican. Analizamos los principios y valores que ordenan las representaciones de la sociedad chilena, además de centrarnos en las dinámicas de clase y género que alimentan las clasificaciones. Este ejercicio permite mostrar que la crítica social y legitimación de las desigualdades pueden operar simultáneamente, aunque en planos distintos.

Palabras clave: desigualdad, crítica, legitimación, sociología pragmática, injusticia. 


\section{Introducción $^{1}$}

Las marcadas desigualdades ${ }^{2}$ socioeconómicas que caracterizan a las sociedades latinoamericanas constituyen uno de sus problemas centrales y han sido bien estudiadas por las ciencias sociales durante la última década. Menos analizados han sido los grados de legitimación ${ }^{3}$ que existen frente a la desigualdad, los cuales pueden representar un mecanismo importante de reproducción de ésta. De hecho, hay una relación entre niveles de igualdad y de legitimación de la desigualdad: mientras las sociedades más igualitarias presentan menor tolerancia a la desigualdad, las sociedades más desiguales tienden a aceptarla y a legitimarla (Markowsky, 1988; Chauvel, 2006).

Esta situación también ha sido comprobada por estudios chilenos que han mostrado que las clases sociales más bajas en la jerarquía social no perciben tan marcadamente la desigualdad como lo hacen otros sectores (Castillo, 2009). Sin embargo, y a pesar de los avances en este campo, no se ha estudiado sistemáticamente lo que ocurre en las otras clases sociales, donde el discurso de la meritocracia habría calado hondo (Garretón y Cumsille, 2002; Espinoza, 2012; Bonnefoy, 2013), al igual que el individualismo agencial (Araujo y Martuccelli, 2014).

1 El presente artículo se inserta en los proyectos Fondecyt 1130276, 1130890, 1140136, COES-CONICYT/FONDAP/15130009. Esta publicación ha sido posible también gracias al financiamiento del Programa de Estímulo a la Excelencia Institucional de la Universidad de Chile a través del Fondo de Fortalecimiento de Productividad y Continuidad en Investigación, Facultad de Ciencias Sociales. Agradecemos el trabajo del equipo, en especial a Oscar Mac-Clure, Ana María Valenzuela, Sergio Ibáñez y a los estudiantes que participaron en la realización del terreno. Agradecemos además los comentarios de Carolina Pinto e Ismael Puga.

2 Si bien el concepto de desigualdad tiene una larga trayectoria teórica en las ciencias sociales, y cuenta con variadas perspectivas, en este artículo utilizaremos una definición en la cual la desigualdad social es entendida como "el resultado de una distribución desigual, en el sentido matemático de la expresión, entre los miembros de una sociedad, de los recursos de ésta" (Bihr y Pfefferkorn, 2008: 9).

3 Si bien existe un amplio debate acerca de la noción de legitimación, adoptamos en el presente trabajo una perspectiva weberiana, construida con base en la noción de legitimidad de la dominación. Siguiendo a este autor, la legitimación es un conjunto de mecanismos que permiten estabilizar la dominación y, por ende, facilitan la aceptación del orden social, basado en valores compartidos en un grupo. Véase Puga (2013). 
Con base en una metodología novedosa, la cual definimos aquí como aproximación pragmática, la presente investigación se ha llevado a cabo a partir de grupos focales que recrean situaciones interactivas de la vida cotidiana. El artículo analiza las modalidades prácticas de calificar y clasificar de personas comunes y corrientes (Boltanski y Thévenot, 1983) en situaciones de interacción e intercambio en un ejercicio cuya meta es la descripción de la sociedad y de los grupos que la integran. Para llegar a una determinada clasificación, criticarla o legitimarla, los actores deben definir y justificar el valor relativo de las personas en la jerarquía social.

Durante el proceso clasificatorio, las personas argumentan y emiten juicios evaluativos de sí mismas y de los demás grupos sociales; esto permite observar la fijación de límites o barreras sociales entre grupos. De esta manera, se hacen visibles los mecanismos de diferenciación subjetiva que construyen y legitiman la desigualdad. Postulamos entonces que a través de las clasificaciones intuitivas y reflexivas que elaboran las personas se puede estudiar cómo se legitiman o cuestionan las desigualdades sociales. También examinamos cuál es la importancia otorgada por los participantes a los atributos personales e individuales y/o a los factores estructurales en la definición de la posición de los sujetos en las distintas categorías que establecieron.

Nos interesamos particularmente por la manera como se declinan ${ }^{4}$ los factores estructurales de clase y género en la dinámica grupal que caracteriza la situación interactiva, así como en las argumentaciones y en las distintas categorías elaboradas por personas comunes y corrientes para describir la sociedad, legitimar su orden o criticarlo. Trabajar con técnicas interactivas permite no sólo atender a la prioridad alcanzada por los discursos hegemónicos que legitiman la desigualdad, sino también analizar los momentos y circunstancias en que se debilita la prioridad otorgada a estos discursos.

En síntesis, en este artículo proponemos ampliar el campo de estudio de las desigualdades sociales para incluir los diversos procesos de legitimación o de crítica a la desigualdad. Afirmamos que los procesos de clasificación muestran que el discurso neoliberal ha sido interiorizado por buena parte de los jugadores; sin embargo, la biografía, las experiencias y las orientaciones morales de las personas llevan a declinar dicho discurso de forma compleja y diversa. Asimismo, concluimos que los sentimientos de malestar llevan a la cristalización de momentos de crítica o ruptura del consenso neoliberal.

4 Expresión usada por Danilo Martuccelli, para tomar distancia crítica de las corrientes que explican de manera lineal y a partir de los factores estructurales los comportamientos de las personas y grupos. La palabra declinar reconoce la capacidad de las personas de dotar de sentido el medio social y del papel de las interacciones cotidianas en la definición, legitimación o crítica de la realidad social (Martuccelli y Lits, 2009). 
En una primera parte, haremos una introducción al campo de investigación donde nos situaremos; luego, en la segunda, revisaremos los antecedentes conceptuales y metodológicos de la investigación. La metodología que se utilizó se describirá en la tercera parte. En las tres secciones siguientes expondremos los resultados de la investigación; primero, los principios y valores que ordenan las presentaciones de la sociedad chilena, el esfuerzo y la educación como criterios de clasificación. Después analizaremos la legitimación de la desigualdad en términos de las dinámicas de género y clase, para cerrar con los principales ejes de crítica a las desigualdades presentes en varios sectores de la sociedad, en momentos de ruptura de significado en los juegos, con el fin de mostrar que crítica y legitimación pueden operar simultáneamente, aunque en planos distintos.

\section{El mandato del esfuerzo propio y de la superación individual}

El establecimiento de un nuevo modelo económico a partir de los años setenta ha dado lugar a cambios importantes no sólo en la gestión productiva, organización del trabajo y en las normativas institucionales, sino también en las prácticas y sistemas de interacción social en la vida cotidiana. En el caso de Chile, el cambio de modelo y su profundización en las décadas siguientes ha significado una nueva forma de racionalidad no sólo de gobierno o en la vida económica, sino también en la vida social e individual (Lechner, 2003; Araujo y Martucelli, 2012), propiciando la conversión del ciudadano en un actor económico que se mueve con independencia del Estado.

En este contexto, las desigualdades se transformarían en un estímulo para que la mayor cantidad de individuos se autorregulen y gestionen sus propios riesgos, incitando a la creatividad y a la competencia, lo que volvería a las desigualdades funcionales a la economía. Este patrón económico está legitimado por un nuevo discurso del capitalismo que tiene como centro la representación de sujetos concebidos como empresarios de sí mismos (Bolstanki y Chiapello, 1999; Foucault, 2006).

En dicho modelo, los individuos reciben el mandato de construir sus biografías de forma autónoma, gracias a sus capacidades, recursos personales, esfuerzo y perseverancia, así como a su habilidad para detectar las oportunidades, de mover redes sociales (Mayol et al., 2013; Araujo y Martuccelli, 2012) y consumir (Aparicio-Cabrera, 2014).

Junto a las persistentes desigualdades estructurales (Tilly, 2000), emergen nuevas desigualdades denominadas "dinámicas", que hacen referencia a la heterogeneidad de situaciones que enfrentan los agentes económicos dentro 
de una misma categoría anteriormente considerada homogénea. Los integrantes de la categoría de asalariados, por ejemplo, no enfrentan situaciones iguales: algunos estarán desocupados, otros tendrán empleos precarios, otros más se ubicarán en puestos subcalificados, lo cual introduce importantes diferencias entre las trayectorias vitales (Fitoussi y Rosanvallon, 2010).

De esta manera, los factores estructurales dejan de ser suficientes para anticipar las trayectorias y para entender las diferencias observadas entre personas. Las antiguas categorías parecen diluirse tras la fuerza de las desigualdades dinámicas que explican las diferencias intracategoriales, por un lado, $\mathrm{y}$ por la interiorización del discurso del esfuerzo, de la perseverancia, del empoderamiento y el autocontrol, por el otro.

En el caso de Chile, este discurso de corte individualista convive en la sociedad no sin tensión con otros discursos políticos y sociales que valoran la igualdad como principio normativo de justicia (Bonnefoy, 2013) e identifican la coexistencia de distintos sistemas de desigualdad. Estos discursos afirman la responsabilidad del Estado de generar, mediante políticas públicas, condiciones para la igualdad de oportunidades y de derechos.

A nivel latinoamericano, las mutaciones anteriormente descritas han despertado el interés de los estudiosos de la estratificación social, quienes siguiendo diversas tradiciones se han abocado a problemas de medición de la estructura social (Filgueira, 2001), cambios en la composición socioeconómica (Franco et al., 2011) y caracterización de los procesos de movilidad social (Espinoza y Núñez, 2014; Espinoza et al., 2013).

Asimismo, ha habido una revitalización de estudios centrados en identidades sociales y particularmente de clase (Méndez, 2008), como una forma de problematizar procesos de cambio sociocultural, resultado de profundos cambios en la estructura social. Especial interés ha suscitado el estudio de las llamadas clases medias, la emergencia y diferenciación de nuevos sectores y su creciente heterogeneidad interna (Méndez, 2008; Franco et al., 2011; Barozet y Espinoza, 2009; Ruiz y Boccardo, 2011).

Otras investigaciones han mostrado que se ha mantenido la distancia entre las clases sociales -aunque haya mejorado heterogéneamente el bienestar y el acceso al consumo en todas las clases-, el aumento de la distancia entre las clases más altas y el resto de ellas y también entre el sector agrario y el no agrario, lo cual significa la vuelta de fenómenos de polarización en los extremos de la estructura social (Espinoza y Núñez, 2014).

Desde el punto de vista de la movilidad social, se constata la existencia de una movilidad a corta distancia, es decir, entre clases sociales contiguas, que se acompaña por el mantenimiento de las fronteras que se erigen entre 
las categorías con mayor poder y el resto de las categorías sociales (Espinoza et al., 2013). Falta, sin embargo, examinar estas mutaciones desde el análisis subjetivo de las desigualdades y su legitimación, punto de anclaje de las mismas en la historia latinoamericana.

\section{De la teoría y los métodos de investigación: el giro pragmático en la legitimación de la desigualdad}

La mayoría de las investigaciones desarrolladas en este campo en Chile y en América Latina durante las últimas dos décadas se ha centrado en las dimensiones estructurales o en las agenciales de la subjetividad. El Proyecto Desigualdades (2009-2012) -dentro del cual surge el presente trabajo- ha intentado propiciar una agenda de estudios de estratificación social que supere dichas dicotomías, que despliegue una aproximación multidimensional ${ }^{5} \mathrm{y}$ articule dispositivos de investigación cuantitativos y cualitativos.

En el marco de ese trabajo, un subequipo del Proyecto Desigualdades llevó a cabo una revisión teórica acerca del estado de la discusión sobre legitimación de la desigualdad ${ }^{6}$ y constató hallazgos que resultaron paradójicos a partir de la realización de la Encuesta Nacional de Estratificación Social (ENES) de 2009: los entrevistados se muestran altamente críticos respecto a la desigualdad en Chile, pero muy poco proclives a apoyar políticas redistributivas que puedan corregir dichas desigualdades.

La revisión del debate y la evidencia recogida señalaron que los individuos tienen juicios críticos sobre la desigualdad, son capaces de dar cuenta del estado actual de la cuestión en sus sociedades, pero a pesar de ello no se involucran en acciones colectivas tendientes a revertir esta situación.

Lo anterior condujo la agenda de investigación hacia la pregunta acerca de los procesos legitimadores de la desigualdad, presentes particularmente en la dimensión situacional o interaccional. Como señalan Schwalbe et al. (2000), desde la tradición pragmática e interaccionista se sostiene que la desigualdad no puede ser entendida de forma separada de los procesos que la legitiman. Así, la legitimación de las desigualdades, incluso de aquellas que aparecen institucionalizadas, dependerá de la interacción cara a cara, por eso ésta debe ser una dimensión clave de su estudio.

5 Las dimensiones tomadas en cuenta en el análisis en este proyecto son edad, género, adscripción a territorios, capital cultural, capital social, ingresos, ocupación, origen étnico.

6 Inspirada fundamentalmente por la visita de Luc Boltanski a Chile en el año 2011 a la Universidad Diego Portales, donde la lectura de sociología pragmática francesa revitalizó la comprensión de estos procesos. 
El presente artículo es tributario de este giro y se inspira tanto teórica como metodológicamente en las investigaciones de Boltanski y Thévenot (1983), en especial en el estudio denominado "Finding one's way in social space: a study based on games". Para ello, adaptamos para Chile una metodología basada en grupos focales diseñada por estos autores e inspirada en la "pragmática del juicio", junto con otros aportes teóricos y metodológicos de la teoría interaccionista (Beetham, 1991; Schwalbe et al., 2000).

Esta aproximación permite entender cómo las personas negocian, confrontan o modifican criterios clasificatorios hasta lograr un consenso en una práctica interactiva que se acerca a la forma como tomamos decisiones y formulamos opiniones en la vida cotidiana.

En efecto, Boltanski y Thévenot (1983) señalan que las personas tienen la habilidad o competencia para reconocer la identidad social de los actores y para manipular los marcadores de esa identidad (manifestada, por ejemplo, en situaciones de confrontación simbólica, desafíos, etc.). Esta habilidad es presentada por la sociología tradicional como una consecuencia inmediata del proceso de socialización (por ejemplo, una disposición dada por el origen de clase) y no como una competencia del actor, por lo que la pragmática del juicio busca entender la construcción de categorías sociales en la interacción, y no a partir de una interpretación de la socialización.

Asimismo, este enfoque asume que el ejercicio de clasificar y categorizar no se hace in abstracto, sino en situaciones particulares, donde confluyen contextos mayores como una determinada estructura social o la circulación de discursos públicos (Cefaï y Terzic, 2012). Las interacciones están simultáneamente situadas en un contexto macro, meso y micro, y este último está caracterizado por relaciones cara a cara, donde categorías, reglas y principios propios del espacio social son ratificados o negociados.

Los participantes en esta investigación, al igual que las personas en la vida cotidiana, adecuan sus criterios anticipando e imaginando las posibilidades de interpretación del otro, y cooperando con los interlocutores en la construcción de una racionalidad común que les permita colaborar o negociar de acuerdo a formas plausibles (Remy y Storrie, 1994; Le Breton, 2008).

Los participantes despliegan competencias de clasificación, estableciendo una relación entre imágenes mentales individuales y procesos públicos/oficiales de representación de grupos. En el proceso de clasificación, los individuos recurren a pares binarios que permitan separar un "ellos" de un "nosotros". En este marco, los aportes de la sociología cultural de Michèle Lamont (1992 y 2014) también fueron fundamentales en la caracterización de dichos procesos de diferenciación. La autora ofrece el concepto de límites/fronteras simbólicas 
y morales, los cuales denotan un ejercicio de clasificación temporal, físico y simbólico que incluye y separa personas, grupos y cosas.

En síntesis, este proyecto es tributario del culturalismo en los estudios de estratificación social influidos por la obra de Bourdieu, pero también ha sido influido por los giros pragmáticos, situacionales y de la sociología cultural americana. De este modo, teoría y metodología han permitido pasar del en qué consisten las desigualdades socioeconómicas en Chile -en sus distintas dimensiones- al desafío de investigar cómo se legitiman las desigualdades, a nivel subjetivo y en situaciones interactivas.

\section{El juego de clasificaciones como soporte de discursos de legitimación y crítica social $^{7}$}

A partir de este referente teórico-metodológico, se construyó un juego de naipes donde cada carta contenía una fotografía e información real de un hombre o mujer de la Región Metropolitana de Chile. Dicha muestra se obtuvo de la Encuesta Nacional de Estratificación Social (2009), atendiendo a un criterio de diversidad socioeconómica y acordando estrictos protocolos de protección de los datos personales.

En cuanto a la dinámica del juego, los participantes - seleccionados según criterios de clase social ${ }^{8}$ como variable principal- fueron organizados en tríos mixtos de acuerdo al grupo ocupacional. Se realizaron seis juegos, con un total de 36 jugadores ${ }^{9}$ (véase la Tabla 1$){ }^{10}$

A cada trío se le entregó un mazo de naipes de 62 cartas; su tarea consistía en clasificarlas de manera colectiva, según las características que consideraran más relevantes, adecuados o legítimos (imagen física, ocupación, ingresos, educación, edad, sexo, lugar de residencia, etnia, religión y nacionalidad para los no chilenos). Más allá de esta consigna general, el equipo no orientó a los jugadores sobre los criterios de clasificación.

7 El detalle de la metodología está disponible en: http://www.desigualdades.cl/wp-content/ uploads/2011/11/Mac-Clure-et-al-Metodolog\%C3\%ADa-juego-de-clasificacionesmayo-2012.pdf.

8 Operativamente, trabajamos con la noción de clase social desarrollada por Erikson y Goldthorpe (1992).

9 Se optó por realizar cuatro tríos representantes de sectores de clases medias, crecientemente relevantes en sociedades como las latinoamericanas: pero se integró de manera comparativa un grupo acomodado y uno del sector popular.

10 Dicha tabla se encuentra en el Anexo, al final del presente artículo (Nota de los editores). 
La primera fase se realizó con tres jugadores acompañados por un moderador. Una vez clasificados los naipes y establecidos los nombres de cada subgrupo de cartas, se solicitó a los participantes señalar cuál era el naipe del montón que mejor representaba a ese grupo. Finalmente, se les solicitó que se refirieran a las distancias y jerarquías sociales entre los grupos (cuán cerca o lejos; o arriba o abajo estaban unos de otros). Una última fase del juego implicó la reunión de dos tríos (seis jugadores que paralelamente estaban llevando a cabo el juego de clasificación en dos tríos) para acordar una clasificación.

Al concluir el juego, se aplicó a cada jugador una entrevista personal, que explora tres espacios: una reflexión sobre las decisiones tomadas, y los sentimientos y reflexiones que las clasificaciones han despertado en el jugador, la vinculación del juego con su propia experiencia biográfica y finalmente una reflexión sobre su percepción de la desigualdad y la injusticia en la sociedad chilena. Los juegos, que duran cada uno cerca de tres horas, fueron registrados en video y significan alrededor de 20 horas de grabación.

Siguiendo la aproximación pragmática descrita en la sección anterior, la tarea configuró una situación de intercambio permanente durante el cual las personas debieron no solamente elegir los criterios de clasificación y argumentar sus propuestas, sino también defender sus puntos de vista, superar desacuerdos y construir consensos para cumplir con la labor asignada. A partir de distintas experiencias, los jugadores - con diferentes edades y recursos- debieron conformar en corto tiempo un colectivo para clasificar los naipes en grupos y posicionarlos en un espacio social.

Para ello, movilizaron marcos cognitivos y valóricos, y recurrieron a la experiencia propia o ajena para fundar sus argumentos. El proceso de clasificación estuvo cruzado de pequeños ejercicios de poder en torno a la definición de liderazgos y la capacidad de incidencia de cada quien sobre los otros, lo que hizo visible la simpatía o rechazo frente a las propuestas. También se expresaron distintas emociones en un clima que, en la mayoría de los casos, fue calmo y distendido. Sólo en situaciones de confrontación abierta se obstaculizó el proceso, dando lugar a clasificaciones defectuosas que se resolvieron en el momento de juntar a los tríos en un solo grupo. ${ }^{11}$

11 Una vez finalizado el juego, todos los participantes, especialmente de procedencia socioocupacional más baja, reconocieron que la situación interactiva los estimuló para pensar y reflexionar sobre su experiencia y a mirar la realidad desde otra perspectiva que la habitual. 
Para examinar la información, se adoptó una perspectiva etnográfica con fuerte análisis situacional, la cual presta atención no sólo a los resultados de la clasificación, sino también a la acción misma de clasificar, a la calidad de las interacciones y a los contenidos de las argumentaciones intercambiadas. ${ }^{12}$

A continuación presentamos los principales resultados de la investigación, haciendo referencia a situaciones ocurridas en cada uno de los tríos. Veremos cómo los criterios para clasificar los grupos encarnan los valores de la ideología neoliberal que subrayan el mérito individual, la capacidad de emprendimiento y esfuerzo personal, y los criterios que legitiman las desigualdades sociales.

Estos criterios compartidos por los diferentes tríos se declinan de distinta manera de acuerdo con la procedencia socio-ocupacional y de género de los participantes. Sin embargo, la adhesión a ellos suele ser contradictoria con la propia experiencia de los participantes. En este sentido, un hallazgo importante son los momentos de cuestionamiento o ruptura del consenso en torno al mérito y esfuerzo que se produce durante la interacción de los participantes en los distintos tríos.

En el acápite siguiente, estos resultados son expuestos en torno a tres ejes de análisis, que permiten no sólo dar cuenta de la forma como las personas perciben la sociedad y la califican, sino también de los mecanismos de legitimación o crítica a las desigualdades.

\section{Principios y valores: el esfuerzo, la responsabilidad individual y la educación como criterios de clasificación}

Durante el juego, cuando los participantes están elaborando la clasificación, la mayoría otorga importancia al esfuerzo y a la responsabilidad personal en la determinación de la posición social de los sujetos, lo que da cuenta de la prevalencia en la sociedad chilena del principio de igualdad de oportunidades por sobre la igualdad de posiciones como principios de justicia social ${ }^{13}$

12 En este artículo, debido a las limitaciones de extensión, se presentan sólo algunos de los juegos y de las entrevistas cuya interacción fue especialmente esclarecedora. Sin embargo, el análisis final considera el conjunto de la información obtenida.

13 La justicia social como componente de la justicia es una construcción a la vez moral y política, tanto individual como colectiva, acerca de lo que es justo en cuanto a derechos, pero también en cuanto a la distribución y redistribución de las ventajas y recursos en la sociedad, sean éstos materiales o simbólicos. 
(Dubet, 2010). Estos valores -fuertemente interiorizados- se constituyen en un principio moral que diferencia a las personas intracategorialmente y a los grupos sociales entre sí.

Desde esta perspectiva, los jugadores distinguen a las personas esforzadas y que se habrían superado, respecto de quienes no habrían aprovechado las oportunidades, resignándose con lo que tenían. Por ejemplo, tanto en el trío 2, conformado por profesores, contadores, técnicos y analistas, como en el trío 5, compuesto por trabajadores independientes y comerciantes, los jugadores coinciden en que la falta de oportunidades de su generación fue superada gracias al esfuerzo personal, la tolerancia al fracaso, los valores de respeto transmitidos por sus padres, y por la ampliación paulatina de oportunidades educativas.

El esfuerzo y el sacrificio presentes en sus narraciones los hace muy críticos frente aquellos que teniendo oportunidades no se sacrificaron ni esforzaron. Por ello, en términos normativos, los participantes erigen una frontera moral que da lugar a una separación recurrente entre "los esforzados" y "los dejados, los flojos"; es decir, quienes según ellos no tienen sentido de superación y aquellos que se esfuerzan para avanzar.

Los logros alcanzados con sacrificio nutren la autoestima, siendo además considerados como un mecanismo indiscutible de movilidad social. Los integrantes de estos tríos destacan sus propias capacidades de emprendimiento, están satisfechos de lo conseguido a pesar de sus bajos ingresos y valoran la posibilidad de que sus hijos hayan ingresado a la universidad.

Trabajador independiente, 54 años: "Los límites están dados por las capacidades de cada persona en hacer o tener ideas para surgir".

Dueña de casa y vendedora, 64 años: "Los esforzados pueden ser técnicos o incluso universitarios, con esfuerzo".

Desde el punto de vista valórico, los integrantes de todos los tríos se autodiferencian, por un lado, de quienes pertenecen a grupos sociales aventajados que desprecian a las personas; y por otro, de quienes no logran salir adelante. Para la mayoría de los participantes en el juego, la movilidad social y personal de corta distancia que han experimentado en sus trayectorias intergeneracionales es atribuida básicamente al esfuerzo propio y familiar, pero también a factores estructurales asociados a la ampliación de oportunidades educativas en las últimas décadas, en especial becas y créditos.

Los logros alcanzados profundizan y generalizan el consenso social acerca de "quien quiere puede" y valida la creencia en la relación causal entre el esfuerzo y el beneficio $\mathrm{Al}$ mismo tiempo, se mantiene la crítica ya conocida a la asistencia recibida del Estado por los grupos más pobres, de quienes se 
sospecha que no hacen los esfuerzos suficientes, precisamente por la ayuda percibida. Esta opinión se encuentra incluso entre los integrantes del grupo más bajo. Un integrante del trío 6 de Trabajadores que tiene educación básica señala lo siguiente:

Hombre, 41 años, pintor: "Que querer es poder [...] En el Chile de hoy, se puede estudiar, no como era antes, e incluso si uno quiere puede llegar a ser presidente [...]. Los de las clases bajas están ahí porque no quieren llegar más lejos".

Sin embargo, durante los juegos y en las entrevistas individuales, los participantes se refieren reiterativamente a los efectos negativos de la desigualdad de oportunidades en sus propias trayectorias: interrupción de estudios, elección de carreras que no respondían a sus motivaciones, ingreso a universidades menos prestigiosas que limitaron su desarrollo profesional futuro.

Los logros alcanzados se basan entonces en atributos personales que contrarrestan los obstáculos: la perseverancia, el no amilanarse ni desfallecer ante los fracasos y en la capacidad de superar las huellas dejadas por historias familiares conflictivas. También se sustenta en la capacidad de protegerse y de avanzar pese a las discriminaciones sufridas, los abusos y la falta de reconocimiento.

Cabe señalar que la exigencia del esfuerzo y la responsabilidad para avanzar es diferente según la procedencia socio-ocupacional de los participantes. La mayoría de los jugadores comienza a clasificar a partir de los naipes que representan a las personas que concentran todas las ventajas, lo que los sitúa sin mayor discusión en la posición jerárquica más alta: estos naipes representan a las personas con mayores niveles de estudio, son profesionales ejecutivos con altos ingresos, tienen mayor capital cultural y viven en las comunas más prestigiadas.

En este caso, los logros obtenidos, la calidad de vida, la estabilidad y seguridad dejan de ser pensadas como productos del esfuerzo personal y pasan a considerarse como el resultado de la posición de privilegio propia y de sus familias. Los jugadores que provienen de posiciones medias -profesionales medios, técnicos o trabajadores que realizan rutinas no manuales y con más intensidad en los sectores independientes y manuales- atribuyen a los integrantes de estos grupos comportamientos moralmente negativos: actitudes de desprecio, de insensibilidad, de frialdad y de maltrato, de abuso frente a personas que no son de su sector social, a quienes no les reconocen sus aportes y sus habilidades. 
Es decir, los jugadores señalan que la justicia de los procedimientos tiene tanto o más valor como la justicia sustantiva ${ }^{14}$ en la evaluación de las posiciones y comportamientos respectivos de los grupos sociales (Mac-Clure y Barozet, 2016). Tal como lo indican otros estudios, consideran que las élites no conocen el desempleo, no sufren los problemas cotidianos y de esta manera encarnan una suerte de desigualdad fundamental (Fitoussi y Rosanvallon, 2010).

Aquí una frontera moral separa el mundo del privilegio sin esfuerzo del mundo del esfuerzo que exige reconocimiento. Por ejemplo, para el trío 5 de Independientes, conformado por dos comerciantes y una dueña de casa, con educación media completa y básica completa, es notoria la incidencia de las personas con más poder, señalando el papel de los empleadores en las situaciones de injusticia y la discriminación en la sociedad chilena. Para este trío, las grandes diferencias no se justifican y se asocian a abusos de los "exitosos".

Hombre, 54 años, comerciante: "Ahora, por un lado, es justo que una persona que se esforzó más, pero nunca una brecha que gane 200.000 a uno que gane 3 millones [de US\$ 320 a US\$ 4850]".

Hombre, 57 años, comerciante: "Es poquísimo; se están aprovechando. Sería mejor ser un temporero con séptimo básico [nivel alcanzado a los 11 años de edad] e irse a trabajar por temporadas [...] Existe un sueldo mínimo, pero la gente no lo respeta".

Dueña de casa y vendedora, 64 años: "Es una barbaridad, ya no les sorprende nada. Muchas veces son las personas que tienen más que se aprovechan de la gente. [...] La mayoría de las personas de clase alta se aprovechan de los demás, haciéndoles trabajar sin pagarles buenos sueldos".

En el grupo de los Trabajadores, una participante señala lo siguiente:

Dueña de casa, 38 años: "Las personas de clase alta han tenido todas las facilidades y comodidades para estar donde están. Por lo tanto, son lo opuesto a la gente de esfuerzo. Ellos vienen con la marraqueta bajo el brazo [...] Porque ellos tenían ya, venían ya, los papás tenían profesión, sus cuestiones, todo. Ellos tienen ya pagadita la universidad, sus cosas. [...] Como que la piel la tuviéramos diferente. Ellos nos ven como un bicho raro".

La crítica moral a los sectores aventajados no significa que los jugadores mencionados no aspiren a que sus hijos se movilicen a posiciones sociales donde obtengan mayores ingresos, adquieran estabilidad y calidad de vida. En el mismo sentido, el valor otorgado al esfuerzo explicaría la elección de representante de los grupos situados en las categorías altas de la jerarquía social.

14 La segunda se refiere a lo que los individuos deberían recibir; mientras la primera especifica los procesos de acuerdo a los cuales se deben distribuir los bienes. 
Los participantes eligen a aquellas personas que en los naipes acceden a este nivel social superior debido a su capacidad de superación y de fuerza personal. Es el caso de la elección del naipe que representa un ingeniero mapuche; y en forma más contradictoria, el caso del naipe de la mujer vicepresidenta de empresa, no por su procedencia social, sino por el hecho de ser mujer, de ser fuerte y tener aspecto de líder de acuerdo con una jugadora.

Adicionalmente, los participantes valoran a las personas que, habiendo experimentado una movilidad social ascendente, no olvidan los valores transmitidos por sus padres y permanecen vinculados a sus grupos de origen; mientras que critican y descalifican a las personas que ascienden, desconociendo sus orígenes y volviéndose petulantes.

Los jugadores también consideraban esforzadas a aquellas personas que realizan tareas muy penosas o que no tienen posibilidades de surgir, debido a su bajo nivel de estudio. Frente a estos grupos, se expresa un sentimiento contradictorio de empatía -especialmente de parte de las mujeres- y de rechazo. Los participantes temen retroceder socialmente a ese mismo nivel y sospechan que posiblemente los sectores más pobres se aprovechen de la asistencia que les otorga el Estado. Dentro de esta categoría, siguiendo dicha argumentación, son elegidos como representante, por ejemplo, una mujer que lava ropa ajena, una mujer que fabrica cepillos de dientes, un temporero, un barredor de feria, o temporeras sin contrato, que según los participantes sí se esfuerzan.

En segundo lugar, el otro criterio elegido por los participantes como forma de estructuración de la sociedad es la educación, en la cual los jugadores depositan expectativas muy altas. Según todos los tríos, la educación permite salir de la pobreza, ascender social y ocupacionalmente, acceder a la cultura para ser tratado igual, acortar distancias sociales y establecer vínculos con personas de otros grupos.

La educación representa también un seguro contra el desempleo y las situaciones laborales precarias. En pos de la educación, se está dispuesto a todos los sacrificios. No obstante, se observa una fisura en esta valoración y expectativa cuando los jugadores reconocen que la educación no es la misma en los colegios fiscales o privados, y no asegura un soporte suficiente para evitar el riesgo de "retroceder".

Para los tríos tres y cuatro, compuestos por secretarias, técnicos y vendedores, la educación es un factor decisivo en el posicionamiento socio-ocupacional; y el bajo nivel educativo es un obstáculo para avanzar en la vida y un motivo de discriminación. En estos tríos, el insuficiente ingreso familiar o situaciones de crisis familiares los obligaron a interrumpir los 
estudios y a resignarse con ingresar a un liceo técnico o acceder a universidades privadas de menor prestigio.

No obstante, para los integrantes de dichos tríos, la educación tiene un doble filo: permite ascenso social a quienes acceden a ella, pero deja a los demás expuestos a la explotación, la discriminación y la desprotección.

Hombre, 40 años, asesor salud: "Estamos fritos, si ahora todo tú postulas o últimamente tú postulas a un trabajo, ¿qué edad tiene? ¿Hasta qué curso llegó? Eeeh octavo [nivel educacional alcanzado a los 12 años]. Ya mira, sabes que necesitamos personal de aseo en tal lado. [...] Y abusan de la persona, porque no tienes la capacidad de reclamar, porque si tú dices: 'Pero oiga, me está pagando 150 y estoy gastado 100 en la locomoción', porque no le dan ni para la locomoción en algunas partes. Entonces búscate en otro lado, nomás cabrito, aquí no estamos pả atender a gente. Si es así, si la educación es un paso, yo creo que lo que más importa, porque puede ser el gallo [tipo] más penca, con la mejor educación, pero tienes educación y te abren las puertas. Sin educación, no te abren ninguna puerta. Lamentablemente es así".

En este sentido, la educación como criterio de clasificación de los naipes en el juego sólo se considera para diferenciar entre los niveles más altos de una escala social. Con distintas denominaciones, los jugadores separan a los profesionales altos de los profesionales medios y luego los técnicos. Bajo el nivel técnico, la educación deja de ser un factor considerado en las clasificaciones, ya que las personas pasan a ser definidas por los oficios que desempeñan, las características de las relaciones laborales y, frecuentemente, por características psicosociales que les son atribuidas (como por ejempla la falta de perseverancia, ser dejados o resignados).

Un bajo nivel educativo es un factor restrictivo en la carrera de la vida, no sólo porque la falta de estudios cierra puertas, sino también porque las habilidades adquiridas por la experiencia que no son certificadas con diplomas son desconocidas y a veces apropiadas por los profesionales de posiciones jerárquicas superiores, desvalorizando y restringiendo la autonomía de los demás trabajadores.

\section{La declinación de clase y género en los procesos clasificatorios}

La lógica del esfuerzo, si bien es compartida por la mayoría de los jugadores, es interiorizada y vivenciada diferentemente según la clase y el género de los participantes: la experiencia biográfica y las orientaciones normativas influyen en la manera en que los jugadores significan los procesos clasificatorios y en la intensidad de las emociones y sentimientos que suscitan las diferencias y distancias sociales. 
En cuanto a la clase social, al trío 1, conformado por profesionales exitosos, le es más fácil entender la tarea, abstraer y argumentar los criterios de clasificación. Sus integrantes también establecen con rapidez normas explícitas para el funcionamiento del trío, lo cual les permite pasar más fácilmente del trabajo individual al colectivo y manejar las diferencias de opinión.

Comienzan clasificando desde los sectores altos y se identifican con las categorías superiores de la jerarquía construida, diferenciándose solamente con las clases altas de mayor poder, que concentran todas las ventajas materiales y simbólicas. En comparación a ellos, a los participantes del resto de los tríos les es más difícil abstraer criterios más generales; al contrario, recurren más a la experiencia personal o ajena y en sus argumentaciones suelen interpretar la información que se les provee de manera más proyectiva, valorando y atribuyendo a las personas representadas en los naipes rasgos psicológicos y una trayectoria de vida.

El tema del conflicto social, la defensa de los intereses propios, el no reconocimiento, el irrespeto, así como el autoritarismo como parte de la reflexión sobre clases sociales, devienen motivos de discusión en todos los tríos, con excepción de aquel de sector más privilegiado. Estos tríos introducen en el debate nuevos temas como los bajos salarios, la pobreza, la presencia de migrantes en el país.

Hombre, 57 años, comerciante independiente: "Hay firmas donde le han quitado los beneficios. Yo no te voy a dar tanto, si te gusta bien, si no te gusta. Así lo hacen. Sabe lo que pasa es que la gente por conservar su trabajo tiene que estar no más, no tiene otro trabajo y no va a estar mirando la calle".

Mujer 64 años, vendedora de catálogo y dueña de casa: "Lo que pasa es que para las asesoras ha sido terrible lo de los peruanos, porque estas niñas como no tienen casa, no tienen comida, entonces ellas pueden [aceptar salarios muy bajos]. Van a comer, tienen su propia comida, su techo, pero claro es una frescura, un aprovechamiento terrible".

Los jugadores comunican las discriminaciones sufridas y los sentimientos asociados; expresan más abiertamente el malestar experimentado, los sentimientos de injusticia, de inseguridad y, sobre todo, el temor continuo de retroceder en lo avanzado. Estos sentimientos se profundizan con la percepción de la distancia infranqueable que los separa de los grupos que concentran todas las ventajas y oportunidades, que, a diferencia de ellos, les permite gozar de la estabilidad y certidumbre en sus vidas.

Por su parte, la pertenencia a un género se intersecta con la procedencia social. Ambas dimensiones están presentes a lo largo del juego, en las dinámicas y alianzas grupales, en la visibilidad otorgada a las mujeres y en el reconocimiento del carácter particular del trabajo del ama de casa. No obstante, la 
pertenencia de género se expresa con mayor intensidad en la dinámica grupal que en los criterios clasificatorios elegidos.

La mayoría de las mujeres no asume posiciones de liderazgo en el proceso de argumentación, manteniendo con menos persistencia sus opiniones durante el juego y tendiendo frecuentemente a conciliar entre criterios opuestos para hacer avanzar la tarea que les ha sido propuesta.

En general, los integrantes del trío empiezan a clasificar los naipes donde se señala el ingreso y la ocupación, y hace a un lado las cartas representando a mujeres sin ingreso, dejándolas juntas bajo el rubro de ama de casa o se las diferenciaba de acuerdo a la educación o el lugar de residencia. En la mayoría de los tríos, se visibiliza el trabajo doméstico y el papel de las mujeres en la educación y proyección futura de sus hijos.

Hombre, 54 años, comerciante: "Si no fuera por las mujeres que se quedan en casa, la sociedad no existiría”.

Mujer dueña de casa y vendedora, 64 años: "La más sacrificada porque uno en la casa trabaja más que nada [...] Y sin sueldo, entonces dependen del marido".

Pero son especialmente las mujeres quienes afirman el valor social de este trabajo y se oponen tanto a las descalificaciones que reduce a las mujeres sin ingresos a la categoría de dependientes o cuando se les percibe que se aprovechan del trabajo de los hombres. Resisten también a las denominaciones que mistifican o banalizan el trabajo doméstico. Son las mujeres en los tríos quienes enfatizan mayoritariamente las consecuencias del papel que le es asignado al género femenino y el sacrificio que les significa, el cual se expresa tanto en la dependencia económica como en la postergación de sus proyectos personales.

$\mathrm{Al}$ respecto, los participantes masculinos, sobre todo de sectores no universitarios, tienen posiciones ambiguas: por un lado, reconocen las contribuciones del trabajo reproductivo, pero también están alertas al posible aprovechamiento de las dueñas de casas de los recursos masculinos, principalmente en el caso de las mujeres con educación.

En suma, la intersección clase/género es especialmente visible en las dinámicas grupales. En la mayoría de los tríos, los comportamientos, las expresiones y entonaciones verbales de las mujeres son menos autoafirmativas que los hombres; a través de expresiones corporales, sonrisas, aceptación de la opinión de los otros, estimulan el desarrollo del juego, bajan la tensión y tienden a conciliar propuestas opuestas. La posición asumida por las mujeres dentro del juego no favorece la emergencia y persistencia de criterios propios, salvo en los casos donde los participantes masculinos emiten juicios negativos o peyorativos acerca de las mujeres. 


\section{El debilitamiento de los consensos y la emergencia de la crítica}

Retomando ahora nuestro análisis desde una perspectiva procesual, podemos señalar que el desarrollo de los juegos durante casi dos horas propició el intercambio permanente de los jugadores, quienes debieron argumentar sus opiniones, negociar sus diferencias y ser capaces de llegar a acuerdos parciales para avanzar en la tarea propuesta. Esto permite analizar no sólo los contenidos de los discursos, sino la manera en que se fueron construyendo los consensos, como también los momentos de ruptura de los acuerdos y de crítica con el contenido del discurso del esfuerzo y responsabilidad personal.

Como hemos visto, por un lado, los tríos consensuaron fácilmente sobre la importancia de la responsabilidad personal y de la educación como motor de avance y un potente medio de adaptación a las prescripciones sociales. Sin embargo, estos acuerdos entraron en tensión frente a algunas opiniones críticas a las asimetrías sociales manifiestas, por ejemplo, en la concentración de privilegios en la élite o en la falta de respeto de los profesionales a otros grupos.

Por otro lado, la referencia a las experiencias personales en los integrantes de los tríos puso en común situaciones compartidas acerca de los obstáculos enfrentados debido al origen social, a la escuela o universidad donde se estudió, por el hecho de tener sólo una carrera técnica o por el barrio donde se vive. Estas situaciones frustrantes que llevaron a los jugadores en ocasiones a renunciar a sus expectativas fueron vividas, según señalan, con sentimientos de exclusión.

Es en estos momentos cuando los participantes se conectaron con experiencias personales negativas referidas a la falta de oportunidades o la falta de respeto hacia ellos por miembros ubicados en las posiciones altas de la jerarquía social, lo cual debilitó al menos momentáneamente la adhesión al discurso dominante del esfuerzo y la responsabilidad individual.

Las maneras de tomar distancia con el discurso neoliberal dominante fue diferente según las clases sociales. En el caso del trío 1 de Servicios altos, se observa primero una adhesión a los principios del modelo neoliberal. Los integrantes del trío mencionado usan criterios multidimensionales para clasificar los naipes de acuerdo la comuna de residencia, nivel de educación, ingreso y prestigio; sus integrantes separan las cartas en cuatro grupos jerárquicamente ordenados: oro, plata, cobre, plomo, inspirados en los tipos de tarjetas bancarias. 
A pesar de la rapidez con la cual se establece la clasificación aparentemente sin disensión entre los jugadores, al terminar el ejercicio emergen algunas reflexiones críticas:

Ingeniera, 32 años: “¿Cachas [Ves] la riqueza? Los que más abundan no tienen riqueza”.

Luego, una terapeuta mayor quiebra el aparente consenso al tildar de deshumanizada, individualista y exitista la clasificación propuesta. Manifiesta su distancia con la rapidez en que clasificaron a las personas, reduciéndolas a un número, a una etiqueta, sin atender, según señala ella, a su condición humana. Sobre esta base, propone nuevos principios para evaluar a las personas que se sustenten en la empatía, la solidaridad y la comprensión de la vulnerabilidad humana.

Terapeuta, 51 años: "Es como difícil para mí clasificar tan fríamente".

Ingeniero, 30 años: "Para mí no es tan difícil porque nosotros en mi trabajo clasificamos por comuna, clase social, nos metemos en los bolsillos de la gente. Es muy distinta una persona que tiene vivir en Maipú aunque tenga un post grado que la que vive en las Condes. Son distintos nichos, distinto es lo que gastan, distinto es lo que arriendan. Puede tener una casa gigante en Maipú y una casa chica en las Condes. ${ }^{15}$ Nosotros trabajamos con datos, somos fríos y uno se acostumbra".

Terapeuta: [...] "Me cuesta ser tan fría".

Ingeniero: "No es frío".

Ingeniera, 32 años: "Tú lo ves de otra cara. Al final todos somos un número, un número de cuánto ganas, cuánto cotizas".

Ingeniero: "Y más en Chile que es una sociedad tan clasista. De a poco se ha ido transformando. Es la sociedad más penosa en ese sentido, te miden por donde estudias, por donde vives, trabajas, cuánto ganas... clasista. Cuando viajas te preguntan por otras cosas, por eso los europeos tienen la mentalidad más abierta”.

Otro tipo de ruptura ocurrió en los tríos no profesionales. En uno de los tríos 3, de Rutinas no manual alta, el participante hombre, una vez revisada las cartas, expone su interpretación sobre la sociedad atravesada por relaciones de poder, de explotación y por la confrontación entre grupos sociales con intereses antagónicos. De acuerdo con su opinión, los sectores más desventajados, sea por la falta de educación, edad u origen campesino, son objeto de discriminación e irrespeto. Para este participante, en la sociedad existen sólo tres grupos: los esforzados, los profesionales dedicados a un trabajo social y el conformado por quienes tienen el poder.

15 Maipú es una comuna de la Región Metropolitana de clase media baja, mientras Las Condes es una comuna de clase alta. 
Las participantes mujeres resisten esta clasificación porque reduce las relaciones humanas a su dimensión de poder y opresión. Argumentan que, según sus experiencias, no todos los profesionales se comportan de una determinada manera, que también pueden ser personas cercanas y tener valores. Le dan más importancia a las cualidades de las personas que a sus mayores recursos, y, por lo tanto, le quitan relevancia a las desigualdades sociales estructurales.

Mujer, técnico computacional, 40 años: "Yo encuentro que L. [otro participante] es tan antisistema y considera que el que tiene plata es mala persona. Con la otra señora, no estábamos de acuerdo porque no siempre es así. Él decía que si tú vives en San Bernardo o La Pintana ${ }^{16}$ no te van a dar trabajo".

Mujer, secretariado técnico, 39 años: "No siempre la gente que gana más sueldo tiene estudios universitarios. No todos los universitarios vienen del sector oriente, ${ }^{17}$ no todos los que pertenecen a los grupos privilegiados son egoístas. [...]. No veo ningún grupo lejano de mi vida diaria, porque yo me puedo relacionar con una persona que no tiene una universidad como con una que es abogado. A lo mejor él no va hablar de leyes pero hablaremos de otra cosa, y lo que no sé lo voy a escuchar y no sepa se lo voy a preguntar. Va todo en tu capacidad mental, al abogado lo puedo mirar de lejos y decir este gallo [tipo] seco, de buen trato, depende de cómo yo me achique en cada caso, lo que permite a la persona ponerme el pie encima. El hecho que los mapuches tengan políticas específicas demuestra que no son discriminados".

En el trío 4 de Rutinas no manuales bajas conformado por técnicos en contabilidad, camareras y recepcionistas, provenientes de familias cuyos padres han sido trabajadores manuales, destaca el rechazo al autoritarismo y la subvaloración de las experticias adquiridas por la experiencia, aunque no sean certificadas.

La dinámica grupal se organiza en torno al significado contradictorio de la educación, las discriminaciones a que están sometidos los sectores sociales bajos, el autoritarismo de las jerarquías en el trabajo y la subordinación en las relaciones laborales. Se critica el no reconocimiento de parte de los profesionales de las habilidades adquiridas en la experiencia que suelen ser más útiles que las adquiridas en la universidad para ejercer ciertos trabajos. En suma, se afirma como valores la autonomía, la no dependencia a un jefe, y la capacidad de riesgo, valores asociados en su discurso a la masculinidad. Otra integrante del trío 4 refuerza esta postura:

16 Comunas de menores recursos de la periferia de Santiago.

17 Comunas más ricas de la Región Metropolitana. 
Mujer, 43 años, recepcionista: "Los apatronados son personas dejadas que no son capaces de dar el paso, mientras que los independientes son seguros, con la capacidad de tomar la decisión e independizarse".

En síntesis, en la mayoría de los tríos, la interiorización del discurso neoliberal de la responsabilidad personal que concibe a los sujetos como empresarios de sí mismos no logra tapar los sentimientos de malestar experimentados por los jugadores a lo largo de sus biografías frente a los obstáculos enfrentados y las discriminaciones sufridas. En distintos momentos del juego, la dinámica grupal ofrece posibilidades de observar críticas a este discurso.

En dichos momentos, los jugadores atisban nuevas alternativas para interpretar las dinámicas sociales; entre otras, el valor de la diversidad de motivaciones y experiencias de vida que conviven en una misma sociedad. Frente a trayectorias orientadas por el éxito individual, los jugadores plantean valores alternativos: el bienestar, la calidad de vida, la realización personal y el reconocimiento de la diversidad de estilos de vida.

\section{Conclusión}

Los resultados expuestos muestran la interiorización del discurso y de los principios neoliberales en los procesos de construcción de grupos sociales diferenciados como forma de representación de la sociedad. En estos espacios de interacción se reproducen las desigualdades de clase y género, al menos. En los actos de nombrar, definir y separar, se establecen fronteras intergrupales que legitiman las desigualdades y las distancias sociales, y además, hacen aceptable la convivencia en un espacio social con lugares prefijados.

La investigación muestra que el grado de interiorización del discurso del esfuerzo y responsabilidad personal se encuentra avalado por la experiencia de movilidad social de corta distancia que muchos han experimentado en Chile.

Sin embargo, la ideología del esfuerzo como factor explicativo de la diferenciación se rompe en al menos dos sentidos. Por una parte, los jugadores consideran que los grupos aventajados tienen privilegios asociados a su posición de origen, lo cual les asegura estabilidad, bienestar y seguridad, independientemente de los esfuerzos personales. Una densa red social y material les protegería de las vicisitudes sociales y económicas.

Por otra parte, para los jugadores es imposible apostar por el esfuerzo en los grupos más desventajados, quienes enfrentan una constelación de factores externos que les impiden avanzar. En este marco, el consenso se fragiliza al ser contrastado con las experiencias de vida de cada quien a la hora de argumentar en la situación interactiva. 
En distintos momentos del juego se debilita la adhesión a los valores neoliberales, sea por la emergencia de discursos que se derivan de marcos cognitivos diferentes o a partir de vivencias compartidas en las experiencias de vida. En el debate, se hicieron presentes, por un lado, ideas que se asocian más bien a la tradición del socialismo, que afirmaron la existencia de fenómenos de dominación y explotación entre las clases sociales; y por el otro, ideas humanistas y cristianas que subrayaron la humanidad común de los seres humanos, la solidaridad y empatía con los vulnerables.

En otros casos, el discurso del esfuerzo y la responsabilidad social no pudo dar cuenta ni justificar las vivencias dolorosas suscitadas por diversas situaciones de discriminación, de irrespeto, que han sufrido aquellas personas al ingresar a nuevos espacios sociales. Saben que los lugares que actualmente ocupan no los protegen de la incertidumbre y riesgo de retroceso, lo cual les suscita malestar.

La satisfacción por los esfuerzos hechos para que sus hijos tengan mayores oportunidades -lo que generalmente se asocia con el ingreso a la universidadhace soportable los sentimientos de irrespeto y frustración de expectativas iniciales. La vivencia de molestia, de sufrimiento y los temores frente a la incertidumbre a lo largo de la vida pueden abrir un espacio al cuestionamiento de las definiciones culturales dominantes que justifican las desigualdades (Varikas, 2005).

De esta manera, una línea de continuidad une la legitimación de la lógica del mérito y la responsabilidad individual con la vivencia cotidiana de desazón ante la desigualdad de oportunidades, constatación de la concentración o acaparamiento de ventajas por parte de los grupos sociales altos, por el irrespeto y, finalmente, por la incertidumbre de perder lo logrado.

\section{Referencias}

Aparicio-Cabrera, Abraham (2014), "Pluralismo moral y bienestar subjetivo del consumidor”, en Convergencia, núm. 64, México: Universidad Autónoma del Estado de México.

Araujo, Kathya y Martuccelli, Danilo (2012), Desafíos comunes. Retrato de la sociedad chilena y sus individuos, Chile: LOM.

Araujo Kathya y Martuccelli, Danilo (2014), "Beyond institutional individualism: Agentic individualism and the individuation process in Chilean society", en Current Sociology, vol. 62, núm. 1, EUA: Sage.

Barozet, Emmanuelle y Espinoza, Vicente (2009), “¿De qué hablamos cuando decimos "clase media"? Perspectivas sobre el caso chileno", en El Arte de Clasificar a los Chilenos, Chile: Expansiva-UDP-La Tercera.

Beetham, David (1991), The legitimation of power, EUA: Palgrave. 
Bihr, Alain y Pfefferkorn, Roland (2008), Le système des inégalités, Francia: La Découverte.

Boltanski, Luc y Thévenot, Laurent (1983), "Finding one's way in social space: a study based on games”, en Social Science Information, vol. 22, núm. 4/5, EUA: Sage.

Boltanski, Luc y Chiapello, Ève (1999), Le nouvel esprit du capitalisme, Paris, Gallimard.

Bonnefoy, Javiera (2013), Justice sociale: De la mobilisation à la mobilité sociale. L'expérience de l'injustice au Chili (1990-2010), Thèse de doctorat en Sociologie, Francia: École des Hautes Etudes en Sciences Sociales.

Castillo, Juan Carlos (2009), “¿Cuál es la brecha salarial justa? Opinión Pública y Legitimación de la Desigualdad Económica en Chile”, en Estudios Públicos, núm. 113, Chile: Centro de Estudios Públicos.

Cefaï, Daniel y Terzic, Cédric [dirs.] (2012), L'expérience des problèmes publics. Perspectives pragmatistes, Francia: Éditions de l'EHESS.

Chauvel, Louis (2006), “Tolérance et résistance aux inégalités”, en Lagrange, H. [ed.], L'épreuve des inégalités, Francia: PUF.

Dubet, François (2010), Repensar la justicia social, Argentina: Siglo XXI.

Encuesta Nacional de Estratificación Social (ENES) (2009), aplicada en el marco del Proyecto Desigualdades (2009-2012), Anillos en Ciencias Sociales SOC 12, "Procesos emergentes en la estratificación chilena: medición y debates en la comprensión de la estructura social", Chile: Conicyt.

Erikson, Robert y Goldthorpe, John (1992), The Constant Flux, Inglaterra: Oxford University Press.

Espinoza, Vicente (2012), “El reclamo chileno contra la desigualdad de ingresos”, en Revista Izquierdas, núm. 12, Chile: Instituto de Estudios Avanzados de la Universidad de Santiago.

Espinoza, Vicente y Núñez, Javier (2014), “Movilidad ocupacional en Chile 2001-2009. ¿Desigualdad de ingresos con igualdad de oportunidades?”, en Revista Internacional de Sociología (RIS), vol. 72, núm. 1, España: Instituto de Estudios Sociales Avanzados.

Espinoza, Vicente et al. (2013), "Estratificación y movilidad social bajo un modelo neoliberal maduro: el caso de Chile", en Revista Lavboratorio, núm. 25, año 14, Argentina: Instituto de Investigaciones Gino Germani.

Fitoussi, Jean-Paul y Rosanvallon, Pierre (2010), La nueva era de las desigualdades, Argentina: Manantial.

Filgueira, Carlos (2001), La actualidad de viejas temáticas: sobre los estudios de clase, estratificación y movilidad social en América Latina, Chile: CEPAL.

Foucault, Michel (2006), Seguridad, territorio, población, Argentina: Fondo de Cultura Económica.

Franco, Rolando et al. [coord.] (2011), Las clases medias en América Latina. Retrospectiva y nuevas tendencias. México: CEPAL-Siglo XXI.

Garretón, Manuel Antonio y Cumsille, Guillermo (2002), "Las percepciones de la desigualdad en Chile”, en Proposiciones, núm. 34, Chile: Sur.

Lamont, Michèle (2014), "Reflections inspired by ethnic Boundary Making: Institutions, Power, Networks", en Ethnic and Racial Studies, vol. 37, núm. 5, Inglaterra: Taylor and Francis Online.

Lamont, Michèle (1992), Money, morals and manners: the culture of the French and American upper-middle class, EUA: University of Chicago Press.

Le Breton, David (2008), L 'interactionnisme symbolique, Francia: PUF. 
Lechner, Norbert (2003), "Los desafíos políticos del cambio cultural”, en Gutiérrez, Paulina y Tomás Moulian [eds.], Norbert Lechner. Obras escogidas, vol. 2, Chile: LOM.

Mac-Clure, Oscar y Barozet, Emmanuelle (2016), "Judgments on (in)justice in a mature neoliberal regime: Results of an empirical game-based research", en Current Sociology, vol. 64, núm. 3, EUA: Sage.

Markowsky, Barry (1988), “Anchoring justice”, en Social Psychology Quarterly, vol. 63, núm. 34, EUA: Sage.

Martuccelli, Danilo y Lits, Grégoire (2009), "Sociologie, Individus, Epreuves. Entretien avec Danilo Martuccelli”, en Emulations, núm. 5, vol. 3, Francia: Presses Universitaires de Louvain.

Mayol, Alberto et al. (2013), El Chile Profundo: Modelos culturales de la desigualdad y sus resistencias, Chile: Liberalia Ediciones y Librería Prosa \& Política.

Méndez, María Luisa (2008), "Middle class identities in a neoliberal age: tensions between contested authenticities", en The Sociological Review, vol. 56, EUA: Wiley Online Library.

Puga, Ismael (2013), The legitimation of social inequalities in Chile. On Legitimacy and ideology, Alemania: Humboldt Universität zu Berlin.

Remy, Jean y Storrie, Tom (1994), Vie quotidienne et démocratie. Pour une sociologie de la transaction sociale, Francia: L'Harmattan.

Ruiz, Carlos y Boccardo, Giorgio (2011), Panorama actual de la estructura social chilena (en la perspectiva de la transformación reciente), Chile: Documento de Trabajo CIES, Universidad de Chile.

Schwalbe, Michael et al. (2000), "Generic Processes in the Reproduction of Inequality: An Interactionist Analysis”, en Social Forces, núm. 79, Inglaterra: Oxford Journals.

Tilly, Charles (2000), Desigualdades persistentes, Argentina: Manantial.

Varikas, Eleni (2005), Lo que no somos. Historicidad del género y estrategias de desidentificación, en Revista Internacional de Filosofía Política, núm. 25, España: Dialnet. 
Anexo

\section{Tabla 1}

\section{Tríos de participantes}

\begin{tabular}{|c|c|c|}
\hline \multicolumn{2}{|c|}{$\begin{array}{l}\text { Trío según clase } \\
\text { social }\end{array}$} & Descripción \\
\hline 1 & $\begin{array}{l}\text { Servicios altos y } \\
\text { Gestión alta }\end{array}$ & $\begin{array}{l}\text { Nivel Socio Económico (NSE): C1; Educación: universitarios, } \\
\text { técnicos profesionales; Actividad: profesionales, empresarios, } \\
\text { independientes con empleados; Ingreso mensual: sobre US\$ } \\
3.300\end{array}$ \\
\hline 2 & $\begin{array}{l}\text { Servicios bajo } \\
\text { y Gestión } \\
\text { baja }\end{array}$ & $\begin{array}{l}\text { NSE: C2-C3; Educación: universitarios y técnicos; Actividad: } \\
\text { profesores, contadores, técnicos, analistas, etc.; Ingreso mensual: } \\
\text { US\$ } 725 \text { a US } \$ 1.150\end{array}$ \\
\hline 3 & $\begin{array}{l}\text { Rutinas no } \\
\text { manuales altas }\end{array}$ & $\begin{array}{l}\text { NSE: C3-D; Educación: técnicos y sin educación superior; } \\
\text { Actividad: secretarias, administrativos; Ingreso mensual: US\$ } \\
415 \text { a US\$ } 825\end{array}$ \\
\hline 4 & $\begin{array}{l}\text { Rutinas no } \\
\text { manuales baja }\end{array}$ & $\begin{array}{l}\text { NSE: C3-D; Educación: técnica y sin educación superior; } \\
\text { Actividad: vendedores, empleados de nivel bajo (junior), } \\
\text { camareras, dependientes de comercio menor; Ingreso mensual: } \\
\text { US } \$ 415 \text { a US } \$ 825\end{array}$ \\
\hline 5 & Independientes & $\begin{array}{l}\text { NSE: C3-D; Educación: técnicos y sin educación superior; } \\
\text { Actividades: comerciantes pequeños, peluqueras, costureras, } \\
\text { jardineros, no asalariados en general; Ingreso mensual: US } \$ 415 \\
\text { a US } \$ 825\end{array}$ \\
\hline 6 & $\begin{array}{l}\text { Trabajadores } \\
\text { manuales } \\
\text { calificados y no } \\
\text { calificados }\end{array}$ & $\begin{array}{l}\text { NSE: D; Educación: estudios básicos o medios incompletos; } \\
\text { Actividades: obreros en general, empleadas domésticas; Ingreso } \\
\text { mensual: US } \$ 415 \text { a US } \$ 500\end{array}$ \\
\hline
\end{tabular}

Fuente: Elaboración propia.

Virginia Guzmán. Doctora en Sociología, subdirectora del Centro de Estudios de la Mujer, Chile. Líneas de investigación: relaciones de género e institucionalidad. Publicaciones recientes: Virginia Guzmán, "Discurso de género e institucionalidad pública en Chile 1980-2010”, en Claudia Mora, Desigualdad en Chile: la continua relevancia del género, Santiago de Chile: Universidad Alberto Hurtado (2013); Virginia Guzmán, Emmanuelle Barozet, Eduardo Candia, Bernardita Ihnen y Bettina Leiva, "Capacidades y género: 
¿Suma o sistema de desigualdades? El caso de Chile”, en Revista CEPAL, núm. 107, Naciones Unidas: Comisión Económica para América Latina y el Caribe (2012); Virginia Guzmán y Claudia Bonan Jannotti, "Políticas de género: modernización del Estado y democratización de la sociedad”, en Sociología y Género, Madrid: Tecnos (2012); Virginia Guzmán, Ute Seibertb y Silke Staabc, Democracy in the Country but not in the Home? Religion, politics and women's rights in Chile, New York: United Nations Research Institute for Social Development (UNRISD), online publication (2010).

Emmanuelle Barozet. Doctora en Sociología, profesora asociada, Universidad de Chile, Chile. Líneas de investigación: estratificación social, clases medias y justicia social. Publicaciones recientes: Emmanuelle Barozet y Vicente Espinoza, "Tout change parce que rien ne change? Politiques sociales et cycles de transformation économique et institutionnelle au Chili”, en Problèmes d'Amérique Latine (en prensa); Oscar Mac-Clure y Emmanuelle Barozet, "Judgments on (in)justice in a mature neoliberal regime: Results of an empirical game-based research", en Current Sociology, núm. 64 (2016); Etienne Penissat, Cécile Brousse, Jérôme Deauvieau, Julien Chevillard, Emmanuelle Barozet, Oscar Mac-Clure, "From statistical categorizations to ordinary categorizations of social space: history and legacy of an original study based on a card game", en Historical Social Research, vol. 41, núm. 2 (2016).

María Luisa Méndez. Doctora en Sociología, profesora asociada, Universidad Diego Portales, Santiago, Chile. Líneas de investigación: aspectos subjetivos de la movilidad y estratificación social; construcción de pertenencia en barrios urbanos; conflictos urbanos. Publicaciones recientes: Nicolás Angelcos y María Luisa Méndez, "Luchas contra la descalificación territorial: movilización por la vivienda digna y defensa del patrimonio", en Latin American Perspectives (en prensa); Modesto Gayo, María Luisa Méndez, Bert Teiltelboim, "Desigualdad social y estructura ocupacional. La terciarización en Chile”, en Cepal Review (en prensa); María Luisa Méndez, "Contesting the Highbrow and Lowbrow Distinction: How Latin American Scholars Engage in Cross-Cultural Debates", en Laurie Hanquinet y Mike Savage, Routledge International Handbook of the Sociology of Art y Culture, New York: Routledge (2015).

Recepción: 10 de junio de 2015.

Aceptación: 17 de mayo de 2016. 